\title{
Age-Related Functional Changes of Prefrontal Cortex in Long-Term Memory: A Repetitive Transcranial Magnetic Stimulation Study
}

\author{
Simone Rossi, ${ }^{1,2}$ Carlo Miniussi, ${ }^{2}$ Patrizio Pasqualetti, ${ }^{3}$ Claudio Babiloni, ${ }^{2,4}$ Paolo M. Rossini, ${ }^{3,5}$ and Stefano F. Cappa ${ }^{6}$ \\ ${ }^{1}$ Dipartimento di Neuroscienze, Sezione Neurologia, Università di Siena, I-53100 Siena, Italy, ${ }^{2}$ Istituto di Ricovero e Cura a Carattere Scientifico S. Giovanni \\ di Dio Fatebenefratelli, I-25125 Brescia, Italy, ${ }^{3}$ Associazione Fatebenefratelli per la Ricerca-Dipartimento di Neuroscienze, S. Giovanni Calibita, \\ Fatebenefratelli Isola Tiberina, I-00186 Roma, Italy, ${ }^{4}$ Dipartimento di Fisiologia Umana e Farmacologia, Università La Sapienza, I-00185 Roma, Italy, \\ ${ }^{5}$ Clinica Neurologia, Università Campus Biomedico, I-00185 Roma, Italy, and ${ }^{6}$ Centro di Neuroscienze Cognitive, Università Salute-Vita S. Raffaele, I-20132 \\ Milano, Italy
}

Neuroimaging findings suggest that the lateralization of prefrontal cortex activation associated with episodic memory performance is reduced by aging. It is still a matter of debate whether this loss of asymmetry during encoding and retrieval reflects compensatory mechanisms or de-differentiation processes. We addressed this issue by the transient interference produced by repetitive transcranial magnetic stimulation (rTMS), which directly assesses causal relationships between performance and stimulated regions. We compared the effects of rTMS (a rapid-rate train occurring simultaneously to the presentation of memoranda) applied to the left or right dorsolateral prefrontal cortex (DLPFC) on visuospatial recognition memory in 66 healthy subjects divided in two classes of age ( $<45$ and $>50$ years).

In young subjects, rTMS of the right DLPFC interfered with retrieval more than left DLPFC stimulation. The asymmetry of the effect progressively vanished with aging, as indicated by bilateral interference effects on recognition performance. Conversely, the predominance of left DLPFC effect during encoding was not abolished in elders, thus probing its causal role for encoding along the life span. Findings confirm that the neural correlates of retrieval modify along aging, suggesting that the bilateral engagement of the DLPFC has a compensatory role on the elders' episodic memory performance.

Key words: long-term memory; neuroimaging; transcranial magnetic stimulation; rTMS; prefrontal cortex; hippocampal formation; aging

\section{Introduction}

Frontal lesions moderately disrupt learning and memory but are not associated with classic amnesia (Alexander et al., 2003), typically caused by bilateral hippocampal damage (Squire et al., 2001). Nevertheless, several neuroimaging studies involving both verbal (Buckner and Wheeler, 2001; Fletcher and Henson, 2001) and visuospatial (Brewer et al., 1998; Kirchhoff et al., 2000; Buckner and Wheeler, 2001) memoranda have indicated an engagement of prefrontal cortex (PFC) areas in episodic memory, with different patterns of activation during encoding and retrieval. Namely, the neuroimaging-based Hemispherical Encoding Retrieval Asymmetry (HERA) theory (Tulving et al., 1994) proposes a functional specialization of the left PFC for encoding and of the right PFC for retrieval. According to its most recent for-

\footnotetext{
Received Feb. 27, 2004; revised July 17, 2004; accepted July 19, 2004.

This work was supported in part by Ministero della Sanità (Progetto Finalizzato 1999) and by Fondazione Telethon Onlus (E.C0985). We thank F. Carducci, F. Babiloni, F. Pauri, C. Fracassi, and C. Busonero for experimental help.

Correspondence should be addressed to Dr. Simone Rossi, Dipartimento di Neuroscienze, Sezione Neurologia, Brain Stimulation and Evoked Potentials Laboratory, Università di Siena, Policlinico Le Scotte, Viale Bracci, I-53100 Siena, Italy. E-mail: Rossisimo@unisi.it.

DOI:10.1523/JNEUROSCI.0703-04.2004

Copyright $\odot 2004$ Society for Neuroscience $\quad$ 0270-6474/04/247939-06\$15.00/0
}

mulation (Habib et al., 2003), the HERA pattern is not absolute and can be affected by the nature of the presented material, as well as by memorization strategies, task difficulty, and item familiarity (Buckner et al., 1999; Wagner et al., 1999; Ranganath et al., 2000; Johnson et al., 2003; Miniussi et al., 2003).

The ability to learn and remember new information declines with aging (Grady and Craick, 2000), and PFC activations in elders tend to be less asymmetric, as conceptualized by the socalled HAROLD [Hemispheric Asymmetry Reduction in OLDer adults (Cabeza, 2002)] model. This model is based on the finding that aging is associated with a reduction of the lateralization in the pattern of PFC activity during cognitive performances, resulting in a loss of the HERA pattern in case of episodic memory. It remains an open question, however, whether asymmetry reductions reflect a compensation mechanism, helping to counteract the age-related cognitive decline, or a de-differentiation process, reflecting the age-related failure to allocate functional resources in a selective manner. Recently, Cabeza et al. (2002) proposed that high-scoring elders, compared with low-performing elders and young subjects, engaged the PFC bilaterally to a functionally useful degree in a source memory task. High-scoring elders also showed preserved left frontal and enhanced right frontal activity 
versus the young during encoding (Rosen et al., 2002). Using event-related functional magnetic resonance imaging, the PFC activity observed during encoding, predicting subsequent successful recognition, was left-lateralized in young subjects but bilateral in older subjects (Morcom et al., 2003).

However, functional neuroimaging data cannot prove the necessary role of PFC activation in episodic memory because an activated area may be simply correlated with, rather than mediating, task performance. Repetitive transcranial magnetic stimulation (rTMS), causing a brief and reversible decline of performance only if the stimulated area is causally engaged in the task (Rossini and Rossi, 1998; Walsh and Cowey, 2000; Walsh and Pascual-Leone, 2003; Rossi and Rossini, 2004), can provide a direct answer to disentangle the compensation/dedifferentiation issue. Hemispheric specializations of the dorsolateral PFC (DLPFC) during long-term memorization have been successfully demonstrated by rTMS (Rossi et al., 2001; Rami et al., 2003; Sandrini et al., 2003; Floel et al., 2004). In particular, we demonstrated that rTMS of the left DLPFC mainly interferes with the encoding of complex pictures and of the right DLPFC with retrieval (Rossi et al., 2001).

Here, we addressed the effects of aging on the lateralization of DLPFC activity in long-term memory using the same rTMS paradigm.

\section{Materials and Methods}

Subjects. Sixty-six healthy right-handed volunteers gave their written informed consent for the study, after the approval of the protocol by the local Ethical Committee. Their medical history and objective neurological examination were normal, and they had never taken neuroactive drugs. No other significant medical conditions were present. Depression was excluded using standardized rating scales.

A first cohort of 24 "young" subjects $<45$ years of age ( 12 males and 12 females) was used to replicate our previous findings (Rossi et al., 2001). After verifying the reproducibility of results (see Fig. 1), the current cohort was pooled with the 13 young subjects from the previous study to allow a reliable regression analysis between functional asymmetries and individual age. Thus, the young group consisted of 37 subjects $(21 \mathrm{fe}-$ males and 16 males; mean age, $29.3 \pm 5.4$ years; range, $20-43$ years), and the "over 50" group consisted of 29 subjects ( 17 females and 12 males; mean age, $61.5 \pm 8.4$ years; range, $50-80$ years). All subjects had at least a high school education. They were studied while comfortably sitting on a reclining chair, with their head stabilized, in front of a 17 -inch monitor. They kept their forearms resting on armchairs, with their right index finger resting between two buttons spaced $6 \mathrm{~cm}$. A 10 min training, performed with a different set of pictures, let the subjects practice with the task and with both sham or active rTMS (both left and right DLPFCs) before the actual experimental session.

Experimental conditions. As in our previous study (Rossi et al., 2001), the experiment included six blocks of encoding, followed by six blocks of retrieval, pseudorandomized and counterbalanced between subjects as order of presentation. For each block of the encoding phase, 16 complex colored magazine pictures (representing eight indoor and eight outdoor scenes) were randomly presented on the monitor for $2 \mathrm{sec}$, with an intertrial interval of $10 \mathrm{sec}$. Images were preceded by a visual warning stimulus (a red spot lasting $1 \mathrm{sec}$ ). Subjects were instructed to press, with their right index finger, one of the two buttons (left, indoor; right, outdoor) after the presentation of a green circle in the middle of each picture (the "go" signal), which appeared $1 \mathrm{sec}$ after the picture presentation.

The six encoding blocks had six corresponding retrieval blocks ( $1 \mathrm{hr}$ later); each one containing 16 pictures of indoor scenes were again presented randomly: eight of them had been seen previously (tests), whereas eight were novel (distractors). The timing of warning and go signals, picture presentation, and intervals were the same as the encoding. Subjects were again asked, in a yes-no recognition task, to answer by pressing one of the two buttons (left, "old" or test; right, distractor) after the go stimulus. The six encoding/retrieval blocks were labeled according to the type (active or sham) and the side (left/right) of the rTMS applied on the DLPFCs (see Table 1 for the complete labeling of the experimental conditions).

Procedures of stimulation. rTMS was delivered through of a focal coil (outer diameter of each wing, $7 \mathrm{~cm}$ ) connected with a standard Mag-Stim Super Rapid stimulator (maximum output, 2.2 Tesla). As detailed previously (Rossi et al., 2001), individual resting excitability thresholds for left and right motor cortex stimulation were first determined by using the same coil and stimulator. The use of individual threshold instead of prefixed intensity of stimulation prevented that anatomo/technical factors attributable to age (i.e., skull thickening, eventual subclinical cortical atrophy) would bias the real efficacy of stimulation. Once the individual thresholds of stimulation were determined, the intensity was reduced by $10 \%$. Thus, the left and right DLPFC were stimulated, when required, with a subthreshold intensity for eventual motor cortex activation (mean intensity of stimulation used, expressed as percentage of the maximal stimulator output: $57.4 \pm 5.8 \%$ for "below 45 " and $61.5 \pm 8.8 \%$ for over 50 subjects, without significant interhemispheric and between-groups differences), which would have overtly interfered with motor actions required for the response. The left and right DLPFCs were stimulated on F3 and F4 (labeling of the 10-20 EEG international system), respectively, according to a previously detailed anatomical localization procedure (Rossi et al., 2001; Miniussi et al., 2003). A mechanical arm maintained the coil in a fixed position. The handle of the coil was angled backward $\sim 45^{\circ}$ away from the midline in that position (marked on the scalp), and its correct positioning was repeatedly checked before the appearance of each warning signal. Trains of 10\% subthreshold rTMS (500 msec, 20 $\mathrm{Hz}$ ) were delivered, when required by the experimental design, simultaneously with picture presentation. The same intensity and timing of rTMS was used for sham stimulation. In this case, the coil was still centered on F3 and F4 but it was held perpendicularly to the scalp surface, so that scalp contact and discharging noise were quite similar to the active stimulation, but the induced magnetic field did not activate cortical neurons (Rossi et al., 2001).

Data analysis. ANOVA for repeated measures was applied to hits-false alarms. Previously, we had used two psychometrics measures ( $\mathrm{C}$ and $\mathrm{d}^{\prime}$ ) (Rossi et al., 2001) deriving from the signal detection theory. However, we observed that many subjects of the present study (mostly belonging to the young group) did not make any false alarms or any recognition error in some conditions (mostly baseline and sham). In this case, $\mathrm{C}$ and $\mathrm{d}^{\prime}$ can be computed only assuming a small proportion of errors, to avoid plusminus infinite values. Because this proportion should be arbitrarily small and the choice could affect the results, we adopted here the traditional "hits" and "hits-false alarms" rates.

The first analysis addressed the differences between the two age groups across the six experimental conditions by means of two-way ANOVA ("condition" as within-subjects factor, "age-group" as between-subjects factor), followed by planned comparisons. In addition, after verifying the differences between groups within control conditions (baseline and 


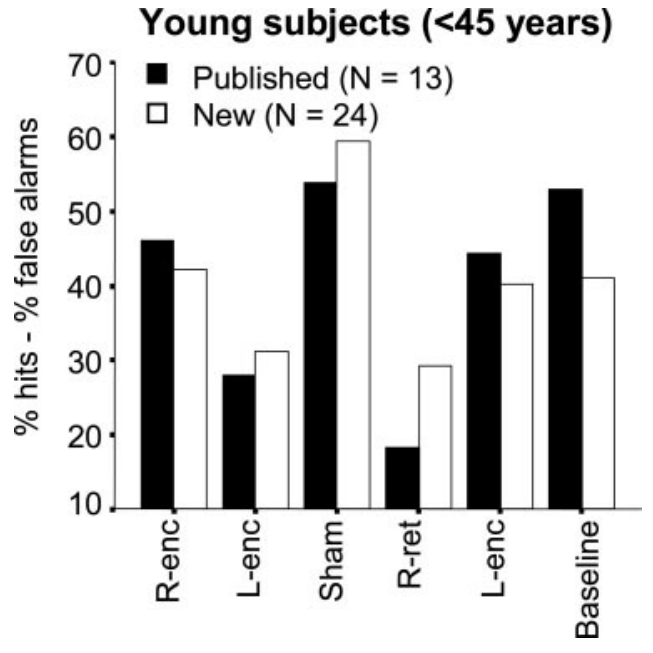

Figure 1. Performance (hits-false alarms) in the previously published and current young subjects groups. The HERA model organization based on left DLPFC functional prevalence in encoding and right in retrieval is fully confirmed. The number of recognition errors in retrieval in the two reference blocks (i.e., baseline and sham) is also similar.

sham), a three-way ANOVA for repeated measures ("hemisphere" and "rTMS stimulation" as within-subjects factors, age-group as betweensubjects factor) was applied to directly address the HERA model and its relationship with age. Finally, two indices of interhemispheric asymmetry were computed as simple differences between hits-false alarms found in R-Enc and in L-Enc [hemispheric encoding asymmetry (HEA)] and between hits-false alarms found in R-Ret and in L-Ret [hemispheric retrieval asymmetry (HRA)]. These two indices were regressed against actual age (not grouped). A curve-fitting algorithm was applied to verify whether higher-order model (quadratic, cubic) could be useful for a significant increase of the explained variance. The level of significance was set at $p<0.05$.

\section{Results}

No side effects of stimulation were reported. rTMS did not evoke muscular responses or saccadic movements in any subject. The previously described HERA pattern (encoding hindered mainly by left stimulation and retrieval strongly interfered by right DLPFC function) (Rossi et al., 2001) was confirmed in a new group of comparable right-handed young subjects. As shown in Figure 1, two similar patterns were found in the two groups of "published" and "new" young subjects ( $<45$ years old). No group $\mathrm{X}$ experimental condition interaction was found $\left(F_{(5,175)}=1.362 ; p=0.241\right)$. In addition, when ANOVA was focused on the HERA hypothesis, with rTMS stimulation (during encoding vs during retrieval) and hemisphere (right vs left) as within-subjects factors and "group" as between-subjects factor (published vs new), no triple interaction was found $\left(F_{(1,35)}=\right.$ $2.019 ; p=0.164)$. The only significant source of variation was rTMS stimulation $\times$ hemisphere $\left(F_{(1,35)}=17.647 ; p<0.001\right)$, thus providing a robust confirmation of our previous findings.

Given these results, the two groups of young subjects were pooled $(n=37)$ and compared with a cohort of over 50 subjects. The first ANOVA (taking into account all the six experimental conditions) indicated a significant age-group main effect $\left(F_{(1,64)}=11.177 ; p=0.001\right)$ and a clear experimental condition $\times$ age-group interaction $\left(F_{(5,320)}=3.565 ; p=0.004\right)$. The differences between the two groups vanished in baseline (planned comparisons; $p=0.492$ ), indicating a similar performance in the absence of any interference (Table 1). No behavioral differences were found in R-Ret ( $p=0.494)$; in this condition, young sub- jects performed even worse than the over 50 subjects, confirming the strong age dependency of the right DLPFC function in retrieval. However, young subjects performed better than old subjects during sham rTMS $(p<0.001)$ (Table 1$)$.

For a better comprehension of the experimental condition $X$ age-group interaction, we defined contrasts between each block and the two control conditions (baseline and sham) in both groups and found the following significant effects (after Sidak correction): in young subjects, L-Enc and R-Ret versus baseline ( $p=0.029$ and $p=0.002$, respectively) and L-Enc, R-Ret, and L-Ret versus sham ( $p<0.001, p<0.001$, and $p=0.022)$; in old subjects, L-Enc and L-Ret versus baseline $(p=0.011$ and $p=$ $0.030)$ without any significant difference versus sham. When considered as a function of age-group, however, the contrast L-Ret versus baseline did not result in an overtly significant difference ( $p=0.063)$. No significant difference was found between sham and baseline neither in young $(p=0.202)$ nor in old ( $p=$ $0.991)$ subjects.

Focusing the ANOVA on hemispheric asymmetries of hitsfalse alarms, the main finding was the significant triple interaction age-group $\times$ rTMS stimulation $\times$ hemisphere $\left(F_{(1,64)}=\right.$ 4.456; $p=0.039$ ) (Fig. 2), in which distinct age-related patterns were clearly identified: in young subjects, hemispheric asymmetries compatible with the HERA model (Rossi et al. 2001) were statistically verified $\left(F_{(1,36)}=15.230 ; p<0.001\right)$. Conversely, functional asymmetries were lost in the older age group $\left(F_{(1,28)}=\right.$ $0.435 ; p=0.515)$.

To address more specifically the effect of age, hemispheric right-left asymmetries during encoding (HEA) and retrieval (HRA) were also separately computed and regressed against the actual subject's age. The polynomial regression analysis did not allow us to identify any relationship between age and HEA $(<1 \%$ of variance was accounted for a linear model, and no significant changes were found with higher-order models). As shown in Figure $3 a$, in the majority of subjects, a positive right-left asymmetry was found, suggesting that, during encoding, a more disrupting effect was observed when rTMS was applied on the left PFC areas with respect to the right ones. In terms of effect size, we found an overall HEA of 12\% (95\% confidence interval, 5-19\%), and the regression line remained almost constantly above the symmetry zero reference line. In other words, a significant and persistent encoding asymmetry (indicated by the consistent higher number of recognition errors after left stimulation) was found in the whole sample, without age-related variations.

In contrast, the factor "age" exerted a significant effect on HRA. A polynomial model with linear and quadratic components accounted for $11.7 \%$ of HRA variance $(p=0.02)$. As shown in Figure $3 b$, the symmetry line (reference value $=0$ ) entered the $95 \%$ confidence interval of the regression curve near the age of 40 and remained within its boundaries up to the highest age values. According to this finding, the functional asymmetry seems to be specific to young subjects more than the functional symmetry typical of the oldest people, $>65$ years of age. However, individual values shown in Figure 3 allowed to observe that, even in the young group, an opposite pattern of asymmetry can be observed eventually.

\section{Discussion}

Imaging studies addressing in vivo the neural mechanisms underlying age-related episodic memory decline are limited. In general, modifications of prefrontal activation in elders, compared with young people, have been reported frequently during encoding and retrieval of both verbal (Cabeza, 1997; Grady et al., 1999; 

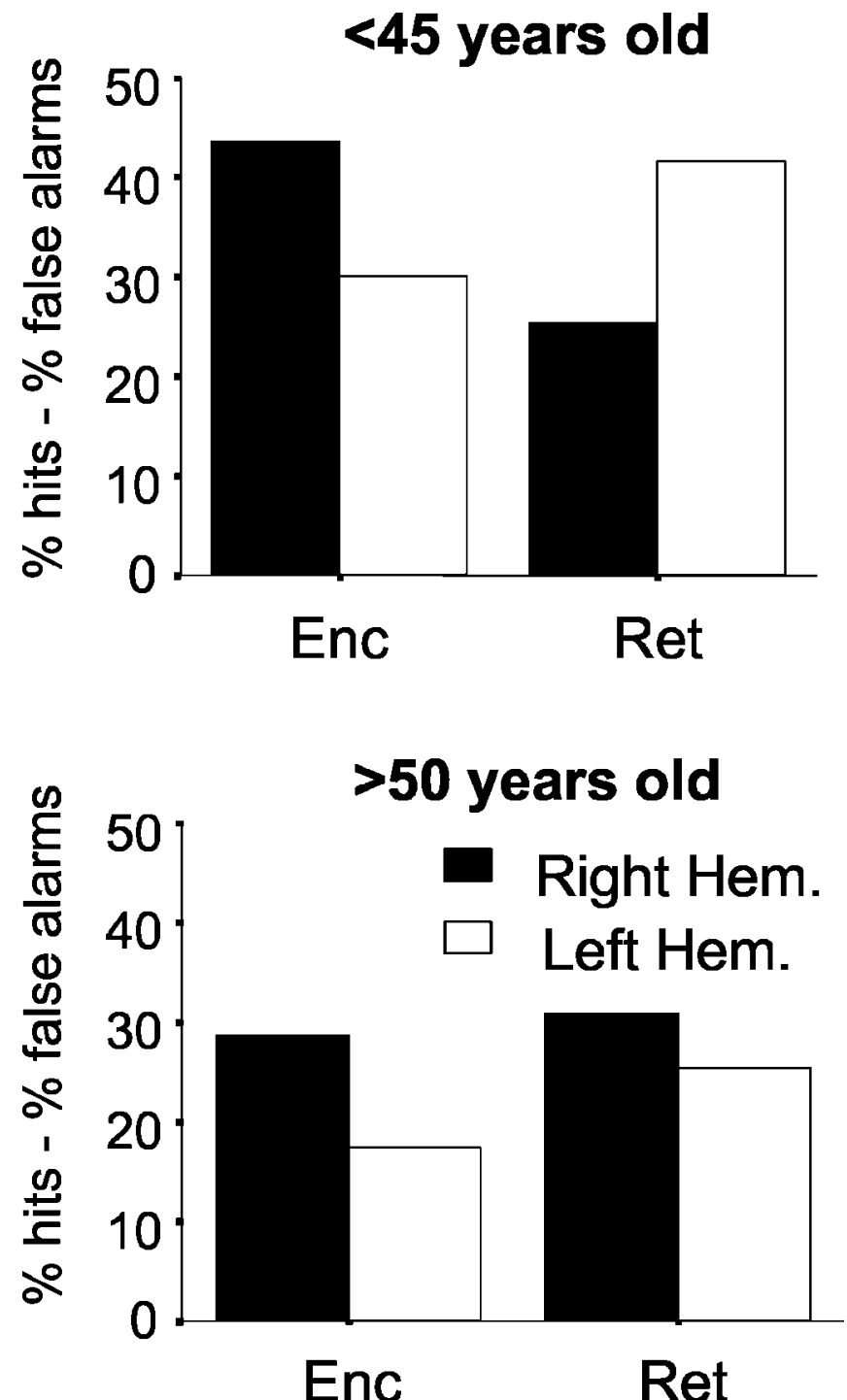

Figure 2. Hemispheric interaction of rTMS effects and age groups during retrieval, according to the HERA model. Top, Young subjects show a clear hemispheric interaction (lower performance during right rTMS in retrieval and when rTMS had been left in encoding). Bottom, In older subjects, the level of performance during active rTMS blocks is generally lower, but the encoding asymmetry is preserved, whereas the retrieval asymmetry is no longer evident. Therefore, the HERA pattern is lost. Note that in old subjects, rTMS of the left DLPFC in retrieval disrupts performance more than rTMS delivered on the right. Statistics are described in Results. Hem., Hemisphere.

Cabeza et al., 2000; Logan et al., 2002; Morcom et al., 2003) and visuospatial (Grady et al., 1995, 1998; Kelley et al., 1998) information. In elders, reduction of left PFC activation has been linked to reduced performance (Stebbins et al., 2002).

The present rTMS results provide direct evidence that the functional role of the PFC in episodic memory undergoes agerelated modifications (Grady and Craick, 2000). Episodic memory performance declines with normal aging (Lamar et al., 2003). Several cognitive factors, such as decline in attentional resources, reduction in processing speed, and defective inhibitory control, have been suggested to be responsible for this decline (Cabeza, 2002). Imaging and electrophysiological studies have assessed the possible underpinnings of these age-associated modifications: in the case of encoding, normal elderly subjects have shown decreased activations in comparison with young subjects in multiple brain regions (Grady et al., 1995; Cabeza, 1997), including the
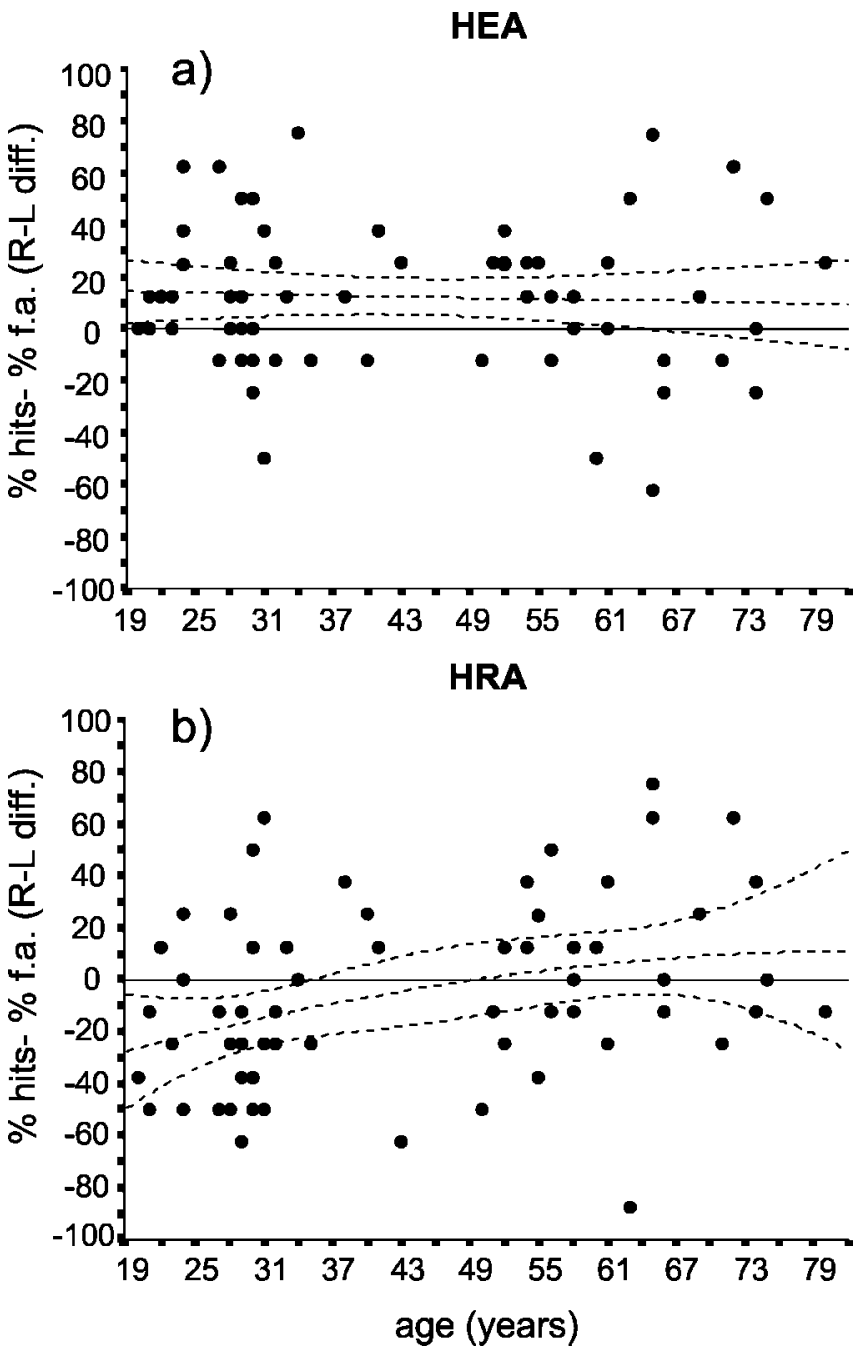

Figure 3. Regressions between the subjects' age and hemispheric right-left asymmetries during encoding (HEA) and retrieval (HRA). a, During the encoding phase, no effect of age was found on hemispheric asymmetries. Indeed, the regression line was constantly above the (thick) zero line, indicating no asymmetry variation across the life span. $b$, During the retrieval phase, in contrast, the age strongly influenced the level of interhemispheric asymmetry, which begins to vanish approximately at 40 years of age. In both panels, some subjects with an opposite pattern of asymmetries can be appreciated (i.e., young without HERA and over 50 with HERA). Statistics are described in Results.

left ventrolateral PFC (Grady et al., 1998), the hippocampus (Grady et al., 1995, 1998; Kelley et al., 1998), and the temporoparieto-occipital regions (Grady et al., 1995; Cabeza, 1997). For retrieval, some studies reported a decline of activation of the right PFC (Cabeza, 1997; Trott et al., 1997), with left PFC compensatory hyperactivation (Cabeza, 1997; Cabeza et al., 2000), whereas others suggest that the brain activity during retrieval is unaffected by aging (Grady et al., 1995; Mark and Rugg, 1998). In these studies, the performance level was generally comparable between young and elderly subjects, thus excluding that the activation differences simply reflect defective memory abilities.

In line with this concept, in the current study, young and old subjects performed similarly in the baseline block. In the sham rTMS condition, young subjects showed a superior performance, suggesting an aspecific alerting effect, probably linked with the auditory stimulation. Behavioral facilitatory effects (i.e., a sort of "priming" effect) in the frame of multisensory stimulations (as auditory clicks in reaction time paradigms) have already been 
described (Nickerson, 1973). This intriguing phenomenon, which needs to be additionally investigated with ad hoc experiments, did not affect the main target of this study (i.e., the level of functional PFC asymmetries), however.

An attempt to integrate neuroimaging findings in episodic long-term memory within a general model of aging effects is the so-called HAROLD model (Cabeza, 2002). According to this hypothesis, normal aging is associated with a reduction of the lateralization in the pattern of PFC activity during cognitive performances. Our findings are in partial agreement with this proposal. At a general level, they indicate that the HERA effect, observed in right-handed young adults for visuospatial material (Rossi et al., 2001), is modified during aging. More specifically, however, we demonstrated, in the same experimental context, differential effects on encoding and retrieval that were not predicted by the HAROLD model. Namely, aging does not influence the role of the left DLPFC during encoding because the left rTMS during encoding consistently decreased the probability of a successful retrieval in both age groups (Fig. $3 a$ ). This points to a clear functional relevance of the left DLPFC for encoding along the life span, at least in this experimental context. Conversely, the predominant role of the right DLPFC during retrieval is age dependent, as indicated by the lack of asymmetry in the effect of the left and right rTMS during retrieval.

The former result must be evaluated in the context of imaging findings that suggested a link between decreased left PFC engagement and the age-related impairment of encoding (Grady and Craick, 2000). It is noteworthy that most studies on aging effects use verbal rather than visuospatial tasks. Right hemisphericdependent processes have been suggested to be particularly sensitive to aging effects (for review, see Dolcos et al., 2002), especially in case of the acquisition and early retrieval of new information (Small et al., 1999a), as in the current study. The persistence of prevalent left rTMS disruption of encoding during aging might thus actually reflect a compensatory increase in the role of the left hemisphere for the encoding of visuospatial material, resulting in a preserved asymmetry. This would suggest that the HAROLD model is hardly applicable to encoding processes of visuospatial material.

The reduction of asymmetry during retrieval follows, prima facie, the HAROLD conceptualization and is amenable to several, nonmutually exclusive, interpretations. Moreover, the present findings help to clarify that the age-related reduction of hemispheric asymmetry may reflect, rather than a de-differentiation process in the right hemisphere, as predicted by the right hemiaging model (Dolcos et al., 2002), a compensatory engagement of the left hemisphere to support a comparable level of performance (Cabeza et al., 2002; Logan et al., 2002). This is a still open question that cannot be solved by traditional neuroimaging investigations, the results of which are merely correlational rather than directly indicating brain regions that mediate task performance (Price et al., 1999). Although with mechanisms that still need to be fully elucidated (Walsh and Cowey, 2000; Walsh and PascualLeone, 2003; Rossi and Rossini, 2004), rTMS interference of a specific region support its causal role in behavioral performance. Thus, the present results are in line with the hypothesis that the additional activations reported in the older subjects, and resulting in loss of asymmetry, have direct functional significance. In particular, the presence of bihemispheric effects of rTMS on memory performance may represent a direct evidence of a compensatory role rather than a de-differentiation mechanism, of the left PFC to support effective retrieval. This concept was put for- ward, but not yet directly demonstrated, in previous studies on high-scoring elderly subjects (Cabeza et al., 2002).

Such evidence, however, does not solve another important question, which is whether compensatory changes could reflect anatomofunctional reorganization or the application of individual, age-related, different cognitive strategies. For example, in working memory tasks, age-related bi-hemispheric activation versus the hemispheric asymmetry of the young has been interpreted as a compensatory mechanism similar to what happens for the execution of simple motor tasks (Babiloni et al., 2000; ReuterLorenz et al., 2000; Smith et al., 2001; Mattay et al., 2002).

Finally, it is necessary to acknowledge that these compensatory changes taking place in the left PFC may be associated with "nonselective recruitment" of additional regions (Logan et al., 2002) that are unaffected by the rTMS of the right DLPFC. Indeed, it is essential to bear in mind that growing evidence highlights the importance of interactions between prefrontal and medial temporal lobe structures during the encoding and retrieval of both verbal and nonverbal material (Buckner and Wheeler, 2001; Simons and Spiers, 2003). A possible candidate for additional recruitment, therefore, is the hippocampal formation, which plays a crucial role in visuospatial episodic memory, especially in the elderly (McIntosh et al., 1999). Although neither hippocampal function nor its possible disruption by prefrontal rTMS can be directly addressed in the frame of this experiment, at least according to the current knowledge of TMS effects on deep brain structures (Walsh and Pascual-Leone, 2003), it can be speculated that in normal aging these circuits could be somewhat potentiated to maintain an acceptable level of performance in episodic visuospatial memory and to compensate the functional decline of the right PFC. In agreement with the prefrontal HAROLD model, bilateral, rather than left-sided hippocampal activation, has been observed in older adults during autobiographical memory retrieval (Maguire and Frith, 2003). Finally, decreased activity of medial temporal structures in elderly individuals is a good predictor of successive cognitive decline (Small et al. 1999b, 2002).

In conclusion, the present study confirms that rTMS is a useful complementary tool to traditional neuroimaging approaches in the investigation of age-associated modifications of the causal engagement of cortical brain regions in human long-term memory. Our results strongly support the hypothesis that retrieval processes in elders is associated with a functional de-specialization of the right DLPFC, with left DLPFC compensation. In contrast, the predominant reliance on the left DLPFC for encoding appears to be preserved along the life span.

\section{References}

Alexander MP, Stuss DT, Fansabedian N (2003) California Verbal Learning Test: performance by patients with focal frontal and non-frontal lesions. Brain 126:1493-1503.

Babiloni C, Babiloni F, Carducci F, Cincotti F, Del Percio C, De Pino G, Maestrini S, Priori A, Tisei P, Zanetti O, Rossini PM (2000) Movementrelated electroencephalographic reactivity in Alzheimer disease. NeuroImage 12:139-146.

Brewer JB, Zhao Z, Desmond JE, Glover GH, Gabrieli JD (1998) Making memories: brain activity that predicts how well visual experience will be remembered. Science 281:1185-1187.

Buckner RL, Wheeler ME (2001) The cognitive neuroscience of remembering. Nat Neurosci Rev 2:624-634.

Buckner RL, Kelley WM, Petersen SE (1999) Frontal cortex contributes to human memory formation. Nat Neurosci 2:311-314.

Cabeza R (1997) Age-related differences in neural activity during memory encoding and retrieval: a positron emission tomography study. J Neurosci 17:391-400.

Cabeza R (2002) Hemispheric asymmetry reduction in older adults: the HAROLD model. Psychol Aging 17:85-100. 
Cabeza R, Anderson ND, Houle S, Mangels JA, Nyberg L (2000) Age-related differences in neural activity during item and temporal-order memory retrieval: a positron emission tomography study. J Cognit Neurosci 12:197-206.

Cabeza R, Anderson ND, Locantore JK, McIntosh AR (2002) Aging gracefully: compensatory brain activity in high-performing older adults. NeuroImage 17:1394-1402.

Dolcos F, Rice HJ, Cabeza R (2002) Hemispheric asymmetry and aging: right hemisphere decline or asymmetry reduction. Neurosci Biobehav Rev 26:819-825.

Fletcher PC, Henson RNA (2001) Frontal lobes and human memory. Insights from functional neuroimaging. Brain 124:849-881.

Floel A, Poeppel D, Buffalo EA, Braun A, Wu CW, Seo HJ, Stefan K, Knecht S, Cohen LG (2004) Prefrontal cortex asymmetry for memory encoding of words and abstract shapes. Cereb Cortex 14:404-409.

Grady CL, Craik IM (2000) Changes in memory processing with age. Curr Opin Neurobiol 10:224-231.

Grady CL, McIntosh AR, Horwitz B, Maisog JM, Ungerleider LG, Mentis MJ, Pietrini P, Schapiro MB, Haxby JV (1995) Age-related reductions in human recognition memory due to impaired encoding. Science 269:218-221.

Grady CL, McIntosh AR, Bookstein F, Horwitz B, Rapoport SI, Haxby JV (1998) Age-related changes in regional cerebral blood flow during working memory for faces. NeuroImage 8:409-425.

Grady CL, McIntosh AR, Rajah MN, Beig S, Craik FIM (1999) The effects of age on the neural correlates of episodic encoding. Cereb Cortex 9:805-814.

Habib R, Nyberg L, Tulving E (2003) Hemispheric asymmetries of memory: the HERA model revisited. Trends Cogn Sci 7:241-245.

Johnson MK, Raye CL, Mitchell KJ, Greene EJ, Anderson AW (2003) fMRI evidence for an organization of prefrontal cortex by both type of process and type of information. Cereb Cortex 13:265-273.

Kelley WM, Miezin FM, McDermott KB, Buckner RL, Raichle ME, Cohen NJ, Ollinger JM, Akbudak E, Conturo TE, Snyder AZ, Petersen SE (1998) Hemispheric specialization in human dorsal frontal cortex and medial temporal lobe for verbal and nonverbal memory encoding. Neuron 20:927-936.

Kirchhoff BA, Wagner AD, Maril A, Stern CE (2000) Prefrontal-temporal circuitry for episodic encoding and subsequent memory. J Neurosci 20:6173-6180.

Lamar M, Resnick SM, Zonderman AB (2003) Longitudinal changes in verbal memory in older adults: distinguishing the effects of age from repeat testing. Neurology 60:82-86.

Logan JM, Sanders AL, Snyder AZ, Morris JC, Buckner RL (2002) Underrecruitment and nonselective recruitment: dissociable neural mechanisms associated with ageing. Neuron 33:1-20.

Maguire EA, Frith CD (2003) Aging affects the engagement of the hippocampus during autobiographical memory retrieval. Brain 126:1511-1523.

Mark RE, Rugg MD (1998) Age effects on brain activity associated with episodic memory retrieval. An electrophysiological study. Brain 121:861-873.

Mattay VS, Fera F, Tessitore A, Hariri AR, Das S, Callicott JH, Weinberger DR (2002) Neurophysiological correlates of age-related changes in human motor function. Neurology 58:630-635.

McIntosh AL, Sekuler AB, Penpeci C, Rajah MN, Grady CL, Sekuler R, Bennett PJ (1999) Recruitment of unique neural systems to support visual memory in normal ageing. Curr Biol 9:1275-1278.

Miniussi C, Cappa SF, Sandrini M, Rossini PM, Rossi S (2003) The causal role of the prefrontal cortex in episodic memory as demonstrated with rTMS. Suppl Clin Neurophysiol 56:312-320.

Morcom AM, Good CD, Frackowiak RS, Rugg MD (2003) Age effects on the neural correlates of successful memory encoding. Brain 126:213-229.
Nickerson RS (1973) Intersensory facilitation of reaction time: energy summation or preparation enhancement? Psychol Rev 80:489-509.

Price CJ, Mummery CJ, Moore CJ, Frackowiak RS, Friston KJ (1999) Delineating necessary and sufficient neural systems with functional imaging studies of neuropsychological patients. J Cognit Neurosci 11:371-382.

Rami L, Gironell A, Kulisevsky J, Garcia-Sanchez C, Berthier M, EstevezGonzalez A (2003) Effects of repetitive transcranial magnetic stimulation on memory subtypes: a controlled study. Neuropsychologia 41:1877-1883.

Ranganath C, Johnson MK, D’Esposito M (2000) Left anterior prefrontal activation increases with demands to recall specific perceptual information. J Neurosci 20:RC108(1-5).

Reuter-Lorenz PA, Jonides J, Smith EE, Hartley A, Miller A, Marshuetz C, Koeppe RA (2000) Age differences in the frontal lateralization of verbal and spatial working memory revealed by PET. J Cognit Neurosci 12:174-187.

Rosen AC, Prull MW, O'Hara R, Race EA, Desmond JE, Glover GH, Yesavage JA, Gabrieli JD (2002) Variable effects of aging on frontal lobe contributions to memory. NeuroReport 13:2425-2428.

Rossi S, Rossini PM (2004) TMS in cognitive plasticity and the potential for rehabilitation. Trends Cogn Sci 8:273-279.

Rossi S, Cappa SF, Babiloni C, Pasqualetti P, Miniussi C, Carducci F, Babiloni F, Rossini PM (2001) Prefrontal cortex in long-term memory: an "interference" approach using magnetic stimulation. Nat Neurosci 4:948-952.

Rossini PM, Rossi S (1998) Clinical application of motor evoked potentials. Electroencephalogr Clin Neurophysiol 106:180-194.

Sandrini M, Cappa SF, Rossi S, Rossini PM, Miniussi C (2003) The role of prefrontal cortex in verbal episodic memory: rTMS evidence. J Cognit Neurosci 15:855-861.

Simons JS, Spiers HJ (2003) Prefrontal and medial temporal lobe interactions in long-term memory. Nat Rev Neurosci 4:637-648.

Small SA, Stern Y, Tang M, Mayeux R (1999a) Selective decline in memory function among healthy elderly. Neurology 52:1392-1396.

Small SA, Perera GM, DeLa Paz R, Mayeux R, Stern Y (1999b) Differential regional dysfunction of the hippocampal formation among elderly with memory decline and Alzheimer disease. Ann Neurol 45:466-472.

Small SA, Tsai WY, DeLa Paz R, Mayeux R, Stern Y (2002) Imageing hippocampal function across the human life span: is memory decline normal or not? Ann Neurol 51:290-295.

Smith EE, Geva A, Jonides J, Miller A, Reuter-Lorenz P, Koeppe RA (2001) The neural basis of task-switching in working memory: effects of performance and ageing. Proc Natl Acad Sci USA 98:2095-2100.

Stebbins GT, Carrillo MC, Dorfman J, Dirksen C, Desmond JE, Turner DA, Bennett DA, Wilson RS, Glover G, Gabrieli JD (2002) Aging effects on memory encoding in the frontal lobes. Psychol Aging 17:44-55.

Squire LR, Clark RE, Knowlton BJ (2001) Retrograde amnesia. Hippocampus 11:50-55.

Trott CT, Friedman D, Ritter W, Fabiani M (1997) Item and source memory: differential age effects revealed by event-related potentials. NeuroReport 8:3373-3378.

Tulving E, Kapur S, Craik FI, Moscovitch M, Houle S (1994) Hemispheric encoding/retrieval asymmetry in episodic memory: positron emission tomography findings. Proc Natl Acad Sci USA 91:2016-2020.

Wagner AD, Koutstaal W, Schacter DL (1999) When encoding yields remembering: insights from event-related neuroimaging. Philos Trans $\mathrm{R}$ Soc Lond B Biol Sci 354:1307-1324.

Walsh V, Cowey A (2000) Transcranial magnetic stimulation and cognitive neuroscience. Nat Rev Neurosci 1:73-79.

Walsh V, Pascual-Leone A (2003) Neurochronometrics of mind: transcranial magnetic stimulation in cognitive science. Cambridge, MA: MIT. 